\title{
Catheter Ablation for Atrial Fibrillation with Heart Failure
}

TO THE EDITOR: In the Catheter Ablation versus Standard Conventional Therapy in Patients with Left Ventricular Dysfunction and Atrial Fibrillation (CASTLE-AF) trial, Marrouche et al. (Feb. 1 issue $)^{1}$ found a $46 \%$ lower rate of death from any cause among patients who were randomly assigned to catheter ablation than among those who were assigned to medical therapy. One concern in interpreting these results is that the patients underwent randomization before the run-in phase. $^{2,3}$

During the run-in phase, 18 patients in the ablation group and 9 in the medical-therapy group were excluded. This process may have affected patient assignments and subverted randomization. Consequent imbalances that may have had an effect on the primary end point include a significantly higher proportion of patients in the medical-therapy group than in the ablation group who had a diagnosis of ischemic cardiomyopathy, who had diabetes mellitus, and who were receiving digoxin at baseline.

Ischemic heart disease, diabetes, and a high serum digoxin concentration have all been associated with increased mortality, and no data regarding digoxin dosing or monitoring were reported in the article. ${ }^{4,5}$ Although ischemic heart disease and digoxin therapy are known confounding factors, the possibility of imbalances of unknown confounding factors cannot be ruled out.

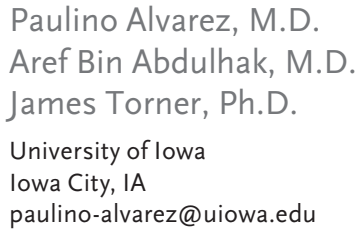

No potential conflict of interest relevant to this letter was reported.

1. Marrouche NF, Brachmann J, Andresen D, et al. Catheter ablation for atrial fibrillation with heart failure. $\mathrm{N}$ Engl J Med 2018;378:417-27.

2. Berger VW, Durkalski VL. Run-in period. In: Wiley StatsRef: statistics reference online. New York: John Wiley \& Sons, 2014 (https://www.wiley.com/learn/wileystatsref/).

3. Roberts C, Torgerson DJ. Understanding controlled trials: baseline imbalance in randomised controlled trials. BMJ 1999; 319:185.

4. Felker GM, Shaw LK, O'Connor CM. A standardized definition of ischemic cardiomyopathy for use in clinical research. J Am Coll Cardiol 2002;39:210-8.

5. Rathore SS, Curtis JP, Wang Y, Bristow MR, Krumholz HM. Association of serum digoxin concentration and outcomes in patients with heart failure. JAMA 2003;289:871-8.

DOI: 10.1056/NEJMc1806519
TO THE EDITOR: In the CASTLE-AF trial, Marrouche et al. found that in patients with heart failure and atrial fibrillation, catheter ablation was associated with a lower rate of death and hospitalization for worsening heart failure than medical therapy. However, the trial design appeared to have structural biases favoring catheter ablation.

Patients were enrolled only if they had had no previous response to or had had unacceptable side effects from antiarrhythmic drugs, or if they were not willing to take these drugs. Such a protocol essentially predetermined a superior outcome with ablation. Also, although they had not had a response to antiarrhythmic agents, patients who were randomly assigned to medical therapy were encouraged to proceed with pharmacologic rhythm control with the use of class IA, class IC, and class III agents, many of which can increase mortality among patients with systolic heart failure. ${ }^{1-4}$

Such probably futile and possibly risky attempts at rhythm control continued in $30 \%$ of the patients in the medical-therapy group. More appropriate approaches would have been to enroll patients regardless of the results of previous antiarrhythmic treatment or to randomly assign patients in whom antiarrhythmic drugs had not worked to catheter ablation or simple rate control.

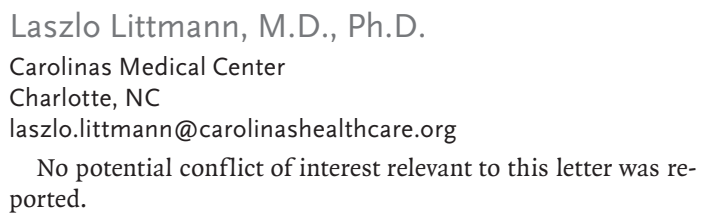

1. Coplen SE, Antman EM, Berlin JA, Hewitt P, Chalmers TC. Efficacy and safety of quinidine therapy for maintenance of sinus rhythm after cardioversion: a meta-analysis of randomized control trials. Circulation 1990;82:1106-16.

2. Echt DS, Liebson PR, Mitchell LB, et al. Mortality and morbidity in patients receiving encainide, flecainide, or placebo the Cardiac Arrhythmia Suppression Trial. N Engl J Med 1991;324: 781-8.

3. K $\phi$ ber L, Torp-Pedersen C, McMurray JJ, et al. Increased mortality after dronedarone therapy for severe heart failure. N Engl J Med 2008;358:2678-87.

4. Freemantle N, Lafuente-Lafuente C, Mitchell S, Eckert L, Reynolds M. Mixed treatment comparison of dronedarone, amiodarone, sotalol, flecainide, and propafenone, for the management of atrial fibrillation. Europace 2011;13:329-45.

DOI: 10.1056/NEJMc1806519

TO THE EDITOR: Marrouche et al. report the superiority of catheter ablation over medical therapy in patients with paroxysmal or persistent atrial fibrillation and a left ventricular ejection fraction 
(LVEF) of 35\% or less who had not had a response to antiarrhythmic drugs, had unacceptable side effects, or were unwilling to take these drugs. The presence of implantable cardioverter-defibrillators (ICDs) indicates that the patients were thought to have irreversible cardiomyopathy while they were receiving target or maximally adjusted doses of guideline-directed medical therapy for heart failure, including beta-blockers.

Notably, in $68 \%$ of the patients in the ablation group, the LVEF improved to $35 \%$ or higher after ablation. Therefore, an astounding number of patients appear to have received ICDs prematurely or inappropriately, even after the exclusion of patients in whom the indication for ICD implantation was secondary prevention and patients receiving cardiac resynchronization therapy. Erroneous implantation due to poor estimation of the adequacy of treatment for atrial fibrillation has been reported in a small series. ${ }^{1}$ The magnitude of erroneous ICD implantation observed in this randomized, controlled trial is alarming.

Current heart-failure guidelines do not systematically address treatment for atrial fibrillation before ICDs are recommended. ${ }^{2}$ The imperative need for data, such as the burden of atrial fibrillation and the target heart rate, to elucidate this nebulous scenario should be a major message from this important trial.

Norman C. Wang, M.D.

University of Pittsburgh Medical Center

Pittsburgh, PA

wangnc@upmc.edu

Dr. Wang reports receiving research support from Boston Scientific and fellowship support from Abbott and Medtronic.
No other potential conflict of interest relevant to this letter was reported.

1. Rodriguez Y, Althouse AD, Adelstein EC, et al. Characteristics and outcomes of concurrently diagnosed new rapid atrial fibrillation or flutter and new reduced ejection fraction. Pacing Clin Electrophysiol 2016;39:1394-403.

2. Yancy CW, Jessup M, Bozkurt B, et al. 2013 ACCF/AHA guideline for the management of heart failure: a report of the American College of Cardiology Foundation/American Heart Association Task Force on practice guidelines. Circulation 2013; 128(16):e240-e327.

DOI: $10.1056 / N E J M c 1806519$

TO THE EDITOR: Marrouche and colleagues report a significantly lower rate of the composite end point with catheter ablation than with medical therapy among patients with atrial fibrillation and heart failure. A substantial number of patients were lost to follow-up in the ablation and medical-therapy groups ( 23 of 179 patients [12.8\%] vs. 10 of 184 patients [5.4\%]). In addition, 28 patients in the ablation group (15.6\%) and 18 patients in the medical-therapy group (9.8\%) did not receive their assigned intervention.

The fragility index, a statistical measure of the robustness of clinical trial results, is the minimum number of events that would have to be added in the group of patients with the smallest number of events in order to change a result from being significant to not significant $(\mathrm{P} \geq 0.05$ by Fisher's exact test). Higher numbers in the fragility index indicate a more statistically robust result. ${ }^{1-3}$ The fragility indexes for the end points reported in the CASTLE-AF trial range from 3 to 11 (Table 1), as compared with a me-

\begin{tabular}{|c|c|c|c|}
\hline Variable & $\begin{array}{l}\text { Ablation } \\
(\mathrm{N}=179)\end{array}$ & $\begin{array}{l}\text { Medical Therapy } \\
(\mathrm{N}=184)\end{array}$ & $\begin{array}{l}\text { Fragility } \\
\text { Index }\end{array}$ \\
\hline Primary end point — no. (\%)† & $51(28.5)$ & $82(44.6)$ & 11 \\
\hline As-treated analysis — no./total no. (\%) & $51 / 169(30.2)$ & $82 / 194(42.3)$ & 3 \\
\hline Per-protocol analysis — no./total no. (\%) & $38 / 129(29.5)$ & $71 / 163(43.6)$ & 3 \\
\hline Death from any cause - no. (\%) & $24(13.4)$ & $46(25.0)$ & 5 \\
\hline Heart failure-related hospitalization — no. (\%) & $37(20.7)$ & $66(35.9)$ & 10 \\
\hline Cardiovascular death — no. (\%) & $20(11.2)$ & $41(22.3)$ & 6 \\
\hline Cardiovascular hospitalization — no. (\%) & $64(35.8)$ & $89(48.4)$ & 4 \\
\hline \multicolumn{4}{|c|}{$\begin{array}{l}\text { * The fragility index, a statistical measure of the robustness of clinical trial results, is the minimum number of events tha } \\
\text { would have to be added in the group of patients with the smallest number of events in order to change a result from } \\
\text { being significant to not significant ( } P \geq 0.05 \text { by Fisher's exact test). Higher numbers in the fragility index indicate a mor } \\
\text { statistically robust result. } \\
\text { The primary end point is a composite of death from any cause or hospitalization for worsening heart failure. } \\
\text { Thetails are available in Table S7 in the Supplementary Appendix of the article, available at NEJM.org. }\end{array}$} \\
\hline
\end{tabular}


dian fragility index of 26 in other trials involving patients with heart failure. ${ }^{2}$ The small fragility indexes, the high proportion of patients lost to follow-up, the greater number of patients lost to follow-up in the ablation group than the fragility index, and the high crossover rates all call into question the validity of the trial findings. Caution should be exercised in translating the results of this trial into clinical practice without further confirmatory evidence.

Kieran F. Docherty, M.B., Ch.B.

Pardeep S. Jhund, M.B., Ch.B., Ph.D.

John J.V. McMurray, M.D.

University of Glasgow

Glasgow, United Kingdom

john.mcmurray@glasgow.ac.uk

No potential conflict of interest relevant to this letter was reported.

1. Walsh M, Srinathan SK, McAuley DF, et al. The statistical significance of randomized controlled trial results is frequently fragile: a case for a Fragility Index. J Clin Epidemiol 2014;67: 622-8.

2. Docherty KF, Campbell RT, Jhund PS, Petrie MC, McMurray JJV. How robust are clinical trials in heart failure? Eur Heart J 2017;38:338-45.

3. Spooner F, Ridgeon EE. Fragility index calculator. 2016 (http://www.fragilityindex.com).

DOI: 10.1056/NEJMc1806519

THE AUTHORS AND A COLLEAGUE REPLY: In response to the critiques by Alvarez et al.: the sensitivity analysis on an intention-to-treat basis with all events included after enrollment yielded a similar hazard ratio $(0.62)$ as those in the perprotocol analysis (0.64) and the as-treated analysis (0.61), with significant $P$ values in each case (Table S7 in the Supplementary Appendix of the article). Although the number of primary endpoint events was higher in patients with ischemic heart failure, those who received digoxin, and those with diabetes, these differences were not significant. Furthermore, as shown in Figure 3 of the article, patients with ischemic cardiomyopathy had a significantly lower event rate with ablation than with medical therapy (35\% vs. $55 \%$; $\mathrm{P}=0.008$ by the chi-square test), which indicates that patients with ischemic cardiomyopathy had a greater benefit from ablation than those with nonischemic cardiomyopathy.

In response to Littmann: until recently, no study had shown that ablation improves hard outcomes in this patient population, including those who have not had a response to antiarrhythmic drugs. Also, the protocol of the CASTLE-AF trial did not mandate the use of antiarrhythmic drugs. Only $28 \%$ of the patients in the medical-therapy group received antiarrhythmic drugs, similar to the proportion in the ablation group (29\%). In fact, most of the patients did not receive any antiarrhythmic drugs before or during the trial.

In response to Wang: patients in our trial had appropriate indications for a dual-chamber ICD or a cardiac resynchronization therapy defibrillator at the time of enrollment. In fact, their indication for ICD implantation was established independently of and before their enrollment in the trial. The improvement in LVEF reported in our trial is not surprising and has occurred in previous trials showing significant improvement in LVEF and dimensions after catheter ablation of atrial fibrillation. Nevertheless, an important lesson learned from the CASTLE-AF trial is to advocate for catheter ablation in this patient population before pursuing device implantation.

In response to Docherty et al.: in the study by Walsh et al., ${ }^{1}$ almost 400 clinical trials with significant results that were published in highimpact journals were evaluated, and the median fragility index in these trials was 8 . A quarter of them had a fragility index of 3 or less, and in more than half of the clinical trials, the number of patients lost to follow-up was greater than the fragility index. In these types of trials, an analysis such as the Cox proportional-hazards model is more suitable, since it takes time into account, whereas the fragility index is a binary analysis with no consideration of time.

Nassir F. Marrouche, M.D.

Mobin Kheirkhahan, M.D.

Comprehensive Arrhythmia Research and Management Center Salt Lake City, UT

nassir.marrouche@carma.utah.edu

Johannes Brachmann, M.D.

Klinikum Coburg

Coburg, Germany

Dr. Kheirkhahan reports no potential conflict of interest relevant to this letter. Since publication of their article, Drs. Marrouche and Brachmann report no further potential conflict of interest.

1. Walsh M, Srinathan SK, McAuley DF, et al. The statistical significance of randomized controlled trial results is frequently fragile: a case for a Fragility Index. J Clin Epidemiol 2014;67: 622-8.

DOI: 10.1056/NEJMc1806519 